\title{
Robot hexápodo para la enseñanza de mecanismos para la transformación de movimientos
}

\author{
Ricardo Rincón Durán ${ }^{1}$ \\ Jorge Armando Niño Vega ${ }^{2}$ \\ Flavio Humberto Fernández Morales ${ }^{3}$
}

Recibido: 10-06-2020

Aceptado: 05-07-2020

\section{Resumen}

En este trabajo se presenta el diseño y aplicación de un prototipo robótico, basado en el movimiento de los hexápodos, como herramienta motivadora para el aprendizaje de mecanismos para transformar movimientos, orientado a estudiantes de la educación media. La investigación tuvo un enfoque mixto, con un diseño cuasiexperimental que involucró a 16 estudiantes de grado décimo, junto con el docente titular del área de tecnología. Es interesante observar que, antes de implementar el prototipo robótico en el aula, los 16 estudiantes tenían dificultades al identificar los conceptos sobre mecanismos; en contraste, luego de haber interactuado con el prototipo durante dos periodos académicos, 15 de los

\footnotetext{
* Artículo de investigación científica y tecnológica derivado de tesis de maestría con enfoque investigativo.

1. Magíster en TIC Aplicadas a las Ciencias de la Educación, licenciado en Tecnología. Docente, Universidad Pedagógica y Tecnológica de Colombia, Sede Duitama, Colombia.

Correo electrónico: ririchter@hotmail.com

ORCID: https://orcid.org/0000-0001-8595-6738

Google Scholar: https://scholar.google.es/citations?hl=es\&user=mQy4fcYAAAAJ
}

2. Magíster en TIC Aplicadas a las Ciencias de la Educación, licenciado en Tecnología. Docente investigador, integrante de los grupos de investigación reconocidos por Colciencias: Grupo de Energía y Aplicación de Nuevas Tecnologías (GEANT) y Didáctica para la Enseñanza de la Ciencia y Tecnología en Niños (DECTEN), Universidad Pedagógica y Tecnológica de Colombia, Sede Duitama, Colombia.

Correo electrónico: jorge.ninovega@gmail.com

ORCID: https://orcid.org/0000-0001-7803-5535

Google Scholar: https://scholar.google.es/citations?hl=es\&user=E_veZbkAAAAJ

3. Doctor en Ingeniería Electrónica, ingeniero electrónico. Docente investigador, director del grupo de investigación avalado por Colciencias GEANT e integrante del grupo DECTEN, Universidad Pedagógica y Tecnológica de Colombia, Sede Duitama, Colombia.

Correo electrónico: flaviofm $1 @$ gmail.com

ORCID: https://orcid.org/0000-0002-8970-7146

Google Scholar: https://scholar.google.es/citations?hl=es\&user $=5$ wOUsekAAAAJ 
16 estudiantes lograron identificar componentes y mecanismos relacionados con la transformación de movimientos. Se concluye que el movimiento de los hexápodos, aplicado en un robot educativo, facilita el aprendizaje de diversas temáticas del área de tecnología, especialmente las relacionadas con los conceptos de mecánica.

Palabras clave: prototipo robótico, enseñanza de mecanismos, transformación de movimientos, hexápodos.

\section{Hexapod robot for teaching mechanisms for transforming movements}

\section{Abstract}

In this work, the design and application of a robotic prototype, based on the movement of the hexapods, is presented as a motivating tool for learning mechanisms to transform movements, oriented at high school students. The research had a mixed approach, with a quasi-experimental design, which involved 16 of tenth grade students, together with the professor of the technology area. It is interesting to note that, before implementing the robotic prototype in the classroom, the 16 students had difficulties in identifying the concepts about mechanisms; In contrast, after having interacted with the prototype during an academic period, 15 of the 16 students identified the components and mechanisms related to the transformation of movements. It is concluded that the movement of the hexapods, applied in an educational robot, facilitates the learning of various topics in the area of technology, especially those related to the concepts of mechanics.

Keywords: robotic prototype, teaching of mechanisms, transformation of movements, hexapods.programming, constructivism, connectivism. 


\section{Introducción}

Los artefactos que se utilizan diariamente están conformados por diversos módulos que garantizan su funcionalidad, tales como: dispositivos eléctricos, electrónicos, software y mecanismos $(\mathrm{He}-$ rrera-Baquero y Prieto-Ortiz, 2018; Mosquera-Mosquera y Ramírez-Martínez, 2020). Estos últimos son los encargados de brindar soporte y movimiento a las estructuras, lo que los hace un objeto de estudio importante en la enseñanza de tecnología (BarreraMesa, Fernández-Morales y Duarte, 2017). La enseñanza de mecanismos para transformar movimientos es una ciencia aplicada que relaciona el análisis cinemático, la geometría y los movimientos de sus componentes (González, Estrada y Roldán, 2016). Uno de los casos de estudio clásico dentro de esta área es el mecanismo de cuatro barras, debido a los diferentes usos que se le pueden dar a las curvas generadas por el acoplador, que permiten su implementación en la realización de proyectos, y propician competencias, habilidades y destrezas en el área de tecnología e informática (González-Campos, Olarte-Dussán y Corredor-Aristizábal, 2017).

La enseñanza de la tecnología plantea la necesidad de desarrollar en los estudiantes la capacidad de usar de manera crítica y creativa el conocimiento adquirido, generando la capacidad para vivir y convivir en grupos cada vez más heterogéneos y un pensamiento autónomo (Tamayo, Zona y Loaiza, 2015; Gutiérrez, 2018). En este sentido, existen varias alternativas para la enseñanza de conceptos científicos y tecnológicos, como los ambientes virtuales de aprendizaje, materiales educativos computarizados, laboratorios remotos y plataformas educativas, por mencionar algunas (Ruiz-Macías y Duarte, 2018; Niño-Vega, Fernández-Morales y Duarte, 2019; Ñáñez-Rodríguez, Solano-Guerrero y Bernal-Castillo, 2019; Tovar-Vergara, 2019). Los prototipos también hacen parte del material didáctico, entre los que se encuentran los llamados robots educativos, los cuales han sido diseñados para reforzar el proceso de enseñanza de conceptos propios de la tecnología (Cárdenas-Martínez, 2019). La robótica educativa es una herramienta de 
aprendizaje en la que se crea el ambiente adecuado de interacción y colaboración para el trabajo en equipo (Márquez y Ruiz, 2014).

En este trabajo se presenta el diseño y aplicación de un prototipo robótico, basado en el movimiento de los hexápodos, como herramienta motivadora para el aprendizaje de mecanismos para transformar movimientos, orientado a estudiantes de la educación media. En la siguiente sección se presenta el fundamento teórico de la investigación, junto con la metodología del proyecto. Luego se describe el prototipo didáctico, así como los resultados de su implementación en el aula. Finalmente, se brindan las conclusiones, destacando la importancia de emplear material didáctico novedoso para la enseñanza de conceptos de tecnología.

\section{Marco teórico y metodología}

\section{Mecanismos para transmisión de movimiento}

- Mecanismo manivela biela: se trata de un mecanismo que transforma un movimiento circular en un movimiento de traslación, o viceversa. Está constituido por un elemento giratorio denominado manivela, conectado a una barra rígida llamada biela, de modo que cuando gira la manivela la biela está forzada a avanzar y retroceder sucesivamente (Rico-Martínez, 2019). Para la construcción de este mecanismo, se toman los parámetros y dimensiones de los elementos que lo componen: la biela debe ser más grande que la manivela, evitando colisiones en el mecanismo, como se muestra en la figura 1.

Figura 1. Mecanismo de manivela biela corredera.

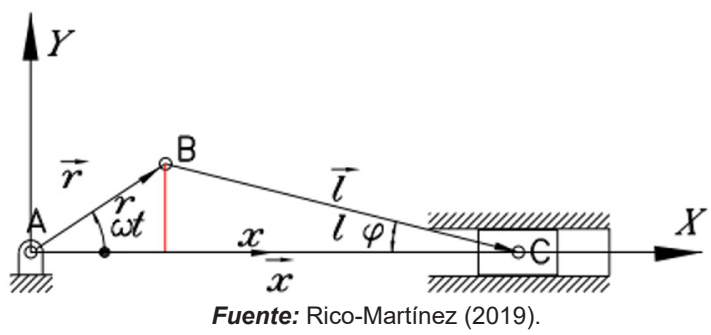


- Mecanismo manivela balancín: los mecanismos articulados de cuatro barras, atendiendo a si alguno de sus elementos puede efectuar una rotación completa, se pueden clasificar en dos categorías; clase l: al menos una de las barras del mecanismo puede realizar una rotación completa (mecanismos de manivela); clase II: ninguna de las barras del mecanismo puede realizar una rotación completa (mecanismos de balancín). El teorema de Grashof proporciona un medio para averiguar la clase a la que pertenece un mecanismo articulado de cuatro barras con solo conocer sus dimensiones y disposición. Si un cuadrilátero no cumple dicho teorema pertenece a la clase II (Machado, Herrera, Roldán y Díaz, 2015).

En un cuadrilátero articulado que cumple el teorema de Grashof, al menos una de sus barras actuará como manivela en alguna de las disposiciones posibles, si se verifica que la suma de las longitudes de las barras mayor y menor es igual o inferior a la suma de las longitudes de las otras dos (Machado et al., 2015). Además, si el soporte del mecanismo es la barra menor, las dos barras contiguas a él actúan de manivelas (mecanismos de doble manivela). Si el soporte del mecanismo es una de las barras contiguas a la menor, la barra menor actúa de manivela y su opuesta de balancín (mecanismos de manivela balancín), como se muestra en la figura 2.

Figura 2. Tipos de mecanismos Grashof.

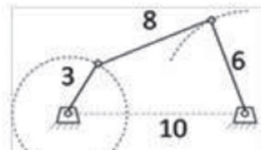

(a) Manivela-balancín

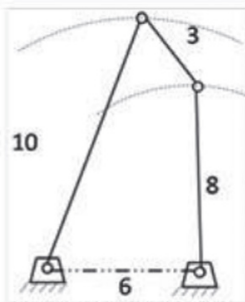

(c) Doble-balancin

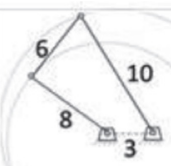

(b) Doble-manivela

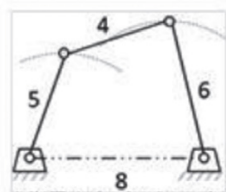

(d) Triple-balancin

Fuente: Machado et al. (2015). 


\section{Prototipo didáctico}

Se denomina prototipo didáctico a todo tipo de material audiovisual, software educativo, modelos tridimensionales y demás material útil en el proceso de enseñanza-aprendizaje, que sirva de apoyo para el logro de los objetivos del plan de estudios de una asignatura (Cabrera, Sánchez, Rojas y Arias-Rojas, 2017). Se trata de un primer modelo que sirve como representación o simulación del producto final, y que permite verificar el diseño y confirmar que cuenta con las características específicas planteadas (Carrera y Ramírez, 2017).

La aplicación de prototipos en el aula permite que los estudiantes puedan adquirir conocimientos de manera eficaz, ya que se puede interactuar con el fenómeno bajo estudio en condiciones amigables (Niño-Vega y Fernández-Morales, 2019). Una ventaja importante es que los prototipos permiten realizar modificaciones necesarias en la fase de desarrollo, mejorando su funcionalidad y diseño para que el recurso educativo tenga una mejor aceptación por parte de los estudiantes.

\section{Estrategia didáctica}

El aprendizaje basado en proyectos (ABP) es una estrategia de integración donde se utiliza un aprendizaje cooperativo centrado en el estudiante, quien construye un conocimiento autónomo en un contexto realista e interesante (Maldonado-Pérez, 2008; AlvisPuentes, Aldana-Bermúdez y Caicedo-Zambrano, 2019). El estudiante también participa en el proceso de valoración, al evaluar el progreso del proyecto o problema, y el desempeño propio, de compañeros y del mentor.

El rol del profesor como mentor y facilitador es uno no tradicional, que busca crear independencia en los estudiantes y aportar con preguntas dirigidas en vez de respuestas, siendo otro inte- 
grante del equipo (Bustamante-Pacari, 2017). En este sentido, para lograr el éxito de estrategias como el ABP es necesario que los docentes cambien la percepción del rol que cumplen en el aula, pasando de la transmisión de conocimientos a la orientación del proceso formativo (Gutiérrez-Rico, Almaraz-Rodríguez y Bocanegra-Vergara, 2019; Jiménez-Espinosa, 2019).

\section{Metodología}

Esta investigación tuvo un diseño cuasiexperimental, con mediación en los ámbitos aplicado y descriptivo, a fin de evaluar el impacto de la robótica educativa aplicada a experimentos de prueba y error, método clave para la creación de prototipos y materiales didácticos (Ordóñez-Ortega, Gualdrón-Pinto y Amaya-Franky, 2019). Se desarrolló bajo un enfoque mixto, cuantitativo y cualitativo, en que se empleó la recolección de datos como base de la medición numérica y el análisis estadístico (Cruz-Rojas, MolinaBlandón y Valdiri-Vinasco, 2019), corroborando la efectividad de la aplicación de prototipos didácticos robóticos.

El trabajo se realizó en la Institución Educativa Sagrada Familia del municipio de Paipa, Boyacá. La muestra estuvo conformada por los estudiantes de grado décimo: ocho hombres y ocho mujeres, con edades de entre 15 y 16 años. También se tuvo el acompañamiento del docente titular de la asignatura, quien brindó su apoyo al proceso investigativo.

La actividad de aula se adelantó durante el tercer y el cuarto periodo académico de 2019 en el área de tecnología e informáti$\mathrm{ca}$, con una intensidad de dos horas semanales. Se establecieron dieciséis sesiones de trabajo, distribuidas así: la primera para aplicar la prueba diagnóstica, las nueve siguientes para desarrollar las diferentes temáticas; las cinco sesiones siguientes se destinaron para la construcción del prototipo robótico por parte de los estudiantes, mientras que en la última sesión se evaluó la construcción del prototipo. 
El desarrollo de los cuestionarios inicial y final se realizó tomando como base los procesos de evaluación de la institución educativa; los lineamientos de desempeño son: superior (4.6-5.0), alto (4.1-4.5), básico (3.5-4.0), bajo (1.5-3.4) y no aprobatorio (0.0-1.4); siendo 3.5 la nota mínima aprobatoria. Los cuestionarios inicial y final se componen de diez preguntas de selección múltiple con una posible respuesta. De ellas, tres están relacionadas a tipos de mecanismos, tres corresponden a componentes de un mecanismo, mientras que las cuatro restantes se basan en cuadriláteros articulados. A cada pregunta del cuestionario se le asigna un valor de 0.5, dando un valor en su sumatoria final de 5.0.

En el desarrollo de la investigación se adelantaron cuatro etapas, a saber:

1. Contempla la aplicación de un cuestionario, donde se evalúan los conocimientos previos de los estudiantes en la temática de mecanismos, identificando las dificultades que presentan los estudiantes en esta área. Con base en los resultados, se establecen los aspectos pedagógicos para la elaboración del prototipo robótico, entre los que se encuentran las temáticas a trabajar.

2. Corresponde al diseño del prototipo robótico, donde se identifican los mecanismos base, aplicándolos para modificar movimientos usando cuadriláteros articulados, según la teoría de curvas de acopladores. El diseño de las piezas se realiza de una manera intuitiva, para que los estudiantes puedan recrearlos sin dificultad.

3. Consiste en el proceso de intervención de la población objeto de estudio con el recurso educativo en el aula de clase. Se busca que los estudiantes apropien los conceptos sobre mecanismos para transformar movimientos, específicamente: manivela biela y manivela balancín, generando curvas con sus 
acopladores y llegando a la construcción del prototipo. En este caso se trabajan los procesos de creación y ensamble, atendiendo al diseño pedagógico del área.

4. Luego de la intervención, se aplica el cuestionario final a los estudiantes, con el propósito de determinar los conocimientos que se adquirieron tras el desarrollo de la experiencia.

\section{Resultados}

A continuación se presentan los resultados obtenidos en cada una de las etapas propuestas en la metodología de esta investigación.

\section{Diagnóstico}

Tras la aplicación del cuestionario inicial, se obtuvo que tres estudiantes poseen un desempeño no aprobatorio y trece estudiantes poseen un desempeño bajo. En cuanto a la identificación de tipos de mecanismos: nueve estudiantes logran identificar mecanismos para modificar fuerzas, quince estudiantes identifican mecanismos para modificar velocidades y seis estudiantes identifican mecanismos para modificar movimientos. Respecto a los elementos existentes en un mecanismo, se obtuvo que tres estudiantes conocen cuántas clases de mecanismos existen, cuatro estudiantes conocen cómo se clasifican los pares cinemáticos y tres estudiantes conocen cómo se clasifican los eslabones. En cuanto a los conocimientos acerca de cuadriláteros articulados, tres estudiantes conocen el mecanismo manivela biela, dos estudiantes identificaron el mecanismo manivela balancín, dos estudiantes identificaron el eslabón acoplador y dos estudiantes identificaron un mecanismo no Grashof.

La prueba inicial permitió evidenciar que la población de estudio posee dificultad en diferenciar los mecanismos de transformación 
de movimientos, específicamente los mecanismos que constan de barras, como lo son: manivela biela y cuadriláteros articulados. Esto justifica el diseño del prototipo didáctico que se describe en la siguiente sección.

\section{Prototipo robótico propuesto}

El prototipo didáctico, correspondiente a un robot hexápodo, permite la movilidad de seis extremidades, emulando el caminar de los escarabajos. Este movimiento se desarrolla a través de la aplicación de mecanismos para la transformación de movimientos, específicamente los mecanismos manivela balancín y manivela biela. El ensamble del prototipo se realiza por medio de simuladores, los cuales permiten evidenciar las trayectorias generadas por los acopladores, encontrar fallas de diseño, problemas mecánicos y errores en el proceso de construcción (Ortega y Sigut, 2016).

En la figura 3 se presenta la estructura propuesta para el diseño del prototipo robótico aplicando mecanismos de manivela biela y manivela balancín. Se destacan seis bloques, que comprenden: el diseño inicial, el análisis, movimientos realizados, optimización del diseño y colisiones de los mecanismos, hasta llegar al diseño óptimo. 
Figura 3. Diagrama de proceso para el diseño de prototipos.

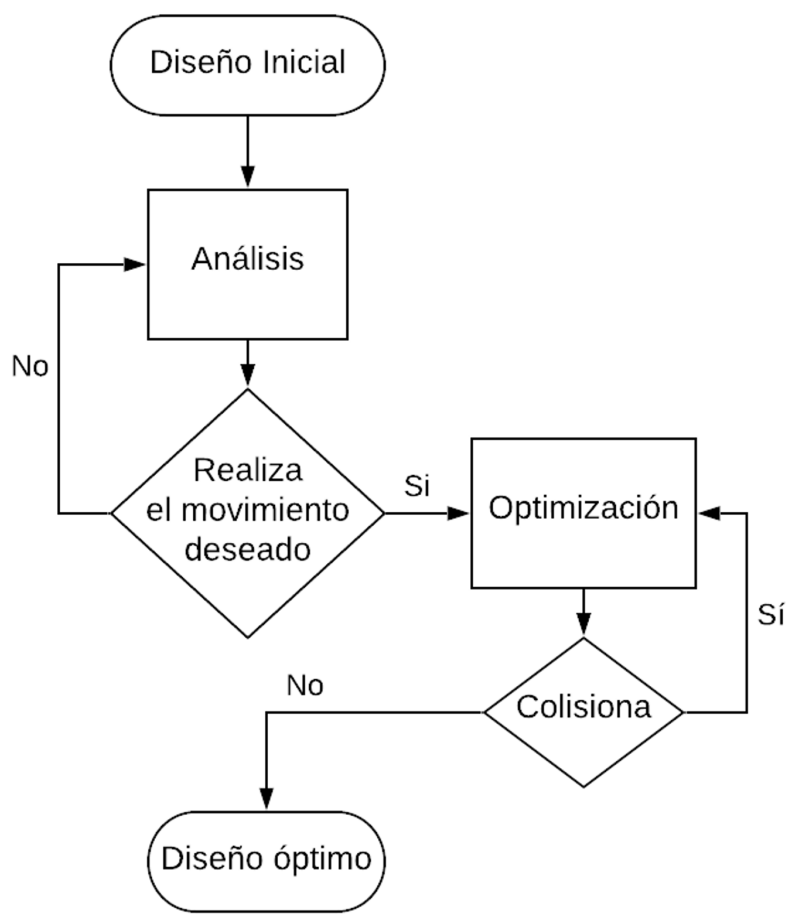

Fuente: elaboración propia.

En el bloque del diseño inicial se estipulan los mecanismos del prototipo robótico, los cuales son: cuadriláteros articulados y mecanismo manivela biela. El bloque de análisis consiste en la posición de elementos de los mecanismos, mientras que en el bloque de realización de movimientos se evalúan y seleccionan las curvas generadas por los acopladores. La optimización consiste en el diseño de los elementos de construcción del prototipo robótico; en la sección de colisiones se tiene en cuenta la posición de elementos correspondientes a los mecanismos bases del prototipo robótico y en el diseño óptimo se presenta el prototipo funcional.

Para el diseño inicial se analiza el sistema morfológico de un escarabajo, tomando como referente el estudio de Martínez (2005). Luego se superpone el mecanismo base manivela balancín imitando la forma base del escarabajo, como se muestra en la figura 4. 
Figura 4. Representación base del mecanismo manivela balancín imitando el movimiento de un escarabajo.

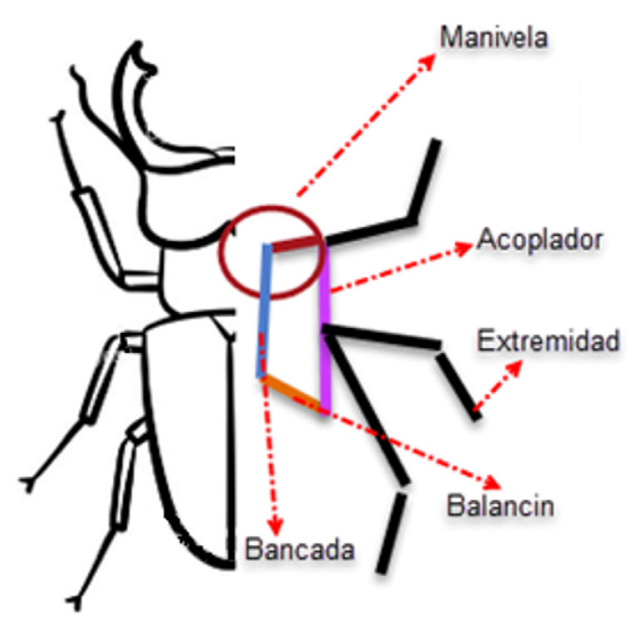

Fuente: elaboración propia.

En cuanto a la construcción del prototipo robótico, se realizaron diez simulaciones hasta llegar al prototipo con diseño óptimo. En la figura 5 se evidencian tres de las simulaciones realizadas a lo largo del diseño del recurso educativo.

Figura 5. Simulaciones del prototipo

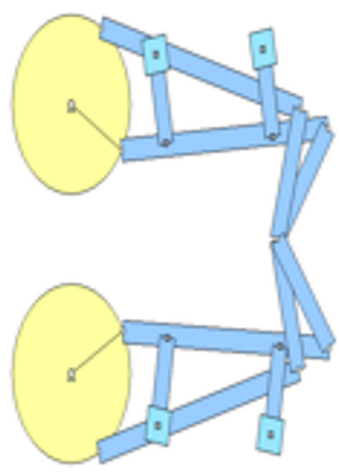

A
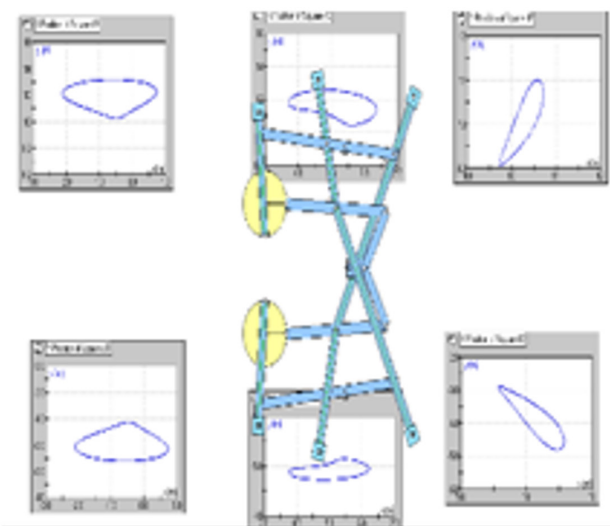

B 


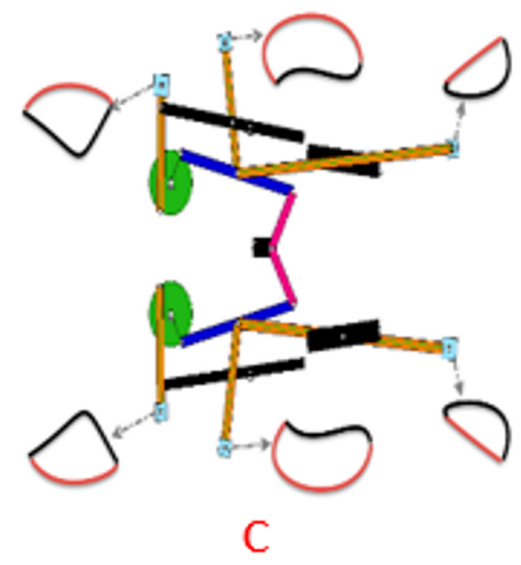

Fuente: elaboración propia.

La sección A de la figura 5 muestra el primer diseño del prototipo, que consta de cuatro mecanismos manivela balancín: tras realizar el análisis, se observó que el mecanismo no puede generar los movimientos deseados. En la sección B se presenta la sexta versión del prototipo; tras el análisis, se observa que realiza los movimientos deseados, pero en la optimización se encuentra que existen colisiones entre sus componentes, lo que dificulta su ensamblaje. La sección $C$ presenta el prototipo final, que cumple con los parámetros de diseño. Este consta de dos mecanismos manivela balancín y dos mecanismos manivela biela, impulsados por dos engranajes.

\section{Experiencia de aula}

El tema a trabajar se presentó por medio de material educativo correspondiente a una serie de textos explicativos que refuerzan los conceptos sobre los tipos de mecanismos existentes, el funcionamiento de los mecanismos de cuatro barras, así como el manejo de herramientas de diseño de mecanismos, entre otros.

En las primeras nueve sesiones se explican los componentes que conforman los mecanismos para la transformación de movimientos. Los temas tratados fueron: tipos de eslabones, cadenas 
cinemáticas, pares cinemáticos, mecanismo manivela biela, cuadriláteros articulados y cómo generar curvas con los acopladores.

Los primeros tres temas se presentaron en una clase magistral, explicando los mecanismos, su funcionamiento, uso y aplicación en diferentes entornos. Los otros temas implican la enseñanza de dos métodos para analizar un mecanismo, a saber: el método gráfico y el analítico. En este sentido, se han implementado diferentes programas en los que el estudiante se puede apoyar para realizar simulaciones, obteniendo valores de desplazamiento, velocidad y aceleración (Torres-Barahona, León-Medina y Pinto-Salamanca, 2017).

En este caso, se implementaron herramientas de simulación como: Geogebra para el reconocimiento de las curvas de los acopladores, planteadas en el Atlas of the four-bar linkage (Todd, Mueller y Fichter, 2014); y Working Model para el desarrollo del montaje virtual de los mecanismos del prototipo. El trabajo de aula implicó una clase magistral para el reconocimiento del entorno de estas herramientas, junto con la presentación de los comandos para realizar el diseño y simulación de los mecanismos de cuatro barras. Luego se desarrollaron ejercicios de construcción de figuras y simulaciones de movimiento de elementos básicos, y se finalizó con una guía de construcción por método gráfico.

En la figura 6, sección A, se presentan los planos que contienen las piezas para ensamblar el prototipo robótico. En la sección $B$ se observa el mecanismo del prototipo, mientras que en la sección $C$ se muestra el prototipo completo. 
Figura 6. Proceso de desarrollo del prototipo.

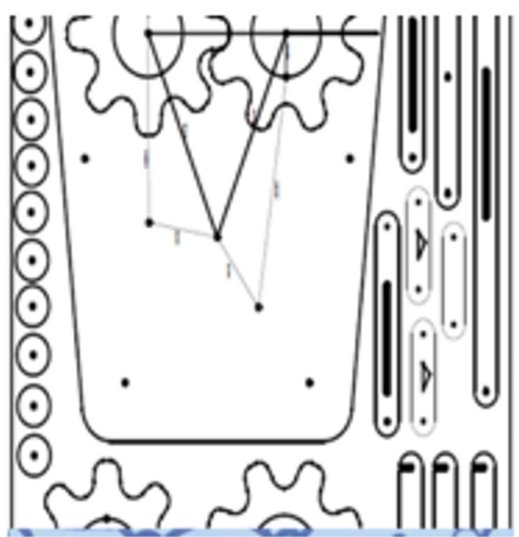

A

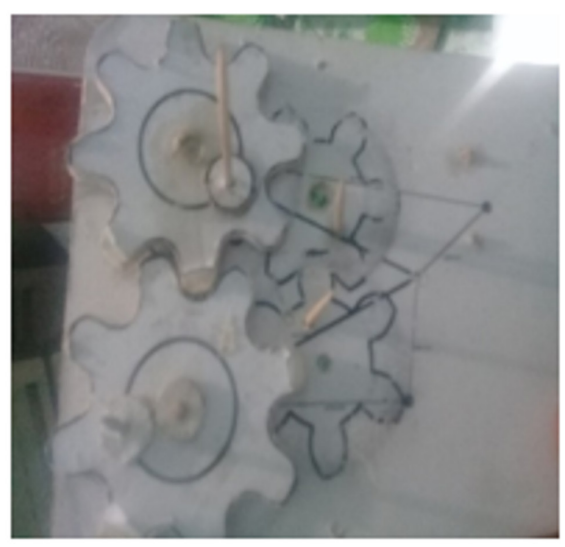

B

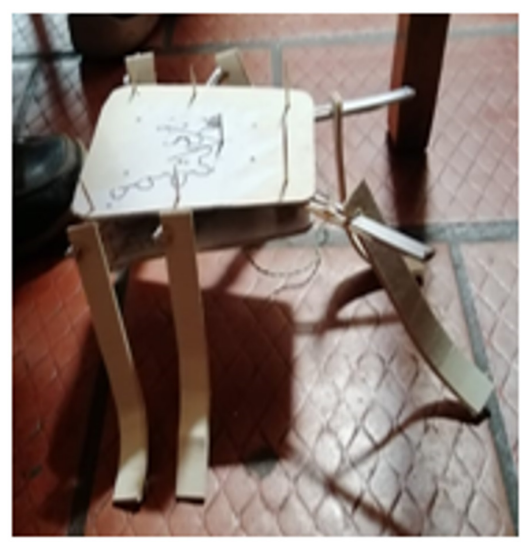

C

Fuente: elaboración propia.

Una vez realizado el diseño en el simulador, se brindan los lineamientos para la construcción del prototipo, de modo que todos los estudiantes participen en su elaboración. El primer lineamiento fue la selección de materiales, que deben ser reciclables. El segundo lineamiento es la elaboración de las piezas de manera individual, dividiendo el proceso en un $50 \%$ en casa y el otro $50 \%$ en clase. El tercer lineamiento es el ensamble del prototipo robótico, como se muestra en la figura 6 , sección B. En la figura 6 , sección $C$, 
se presenta la entrega del prototipo robótico, en donde el robot se encuentra ensamblado y realiza los movimientos deseados.

La construcción del prototipo robótico se realizó por grupos, organizados así: seis grupos de dos estudiantes, un grupo formado por tres estudiantes y un grupo formado por un estudiante, esto para la elaboración de piezas y ensamble del prototipo con ayuda del investigador. En la tabla 1 se muestra la rúbrica para evaluar el prototipo, de acuerdo con el ensamble y funcionalidad de los mecanismos.

Tabla 1. Rúbrica para la evaluación del prototipo.

\begin{tabular}{|l|l|c|}
\hline Ítem & Calificación & Grupos \\
\hline $\begin{array}{l}\text { Presentación del prototipo funcional y realiza los } \\
\text { movimientos planteados }\end{array}$ & $4.5-5.0$ & 2 \\
\hline Presentación del prototipo sin ensamble de las extremidades & $4.0-4.4$ & 4 \\
\hline $\begin{array}{l}\text { Presentación de la construcción de los mecanismos bases } \\
\text { funcionales del prototipo }\end{array}$ & $3.5-3.9$ & 1 \\
\hline Presentación de las piezas de ensamble sin ensamblaje & $2.0-3.4$ & 0 \\
\hline No presenta ni evidencia la construcción del prototipo & $0-1.9$ & \\
\hline
\end{tabular}

Fuente: elaboración propia.

En cuanto a los resultados, dos grupos - uno de dos y otro de un estudiante- presentaron la construcción del prototipo funcional, permitiendo evidenciar el caminar de un robot móvil. Cuatro grupos de dos estudiantes presentaron la construcción del prototipo, pero sin ensamblar las extremidades, permitiendo evidenciar que se generan las curvas esperadas en los acopladores. Un grupo de dos estudiantes presentó el ensamble de los mecanismos manivela biela y manivela balancín explicando el funcionamiento de cada una de las piezas y cómo se ensamblaron para evitar colisiones. El grupo de tres estudiantes presentó las piezas del prototipo sin ensamblar, describiendo el funcionamiento de cada elemento por separado. 


\section{Prueba final}

En la figura 7 se presenta una comparación del desempeño alcanzado por los estudiantes en las pruebas inicial y final. Es notoria la mejoría después de que los estudiantes realizaron la construcción del prototipo robótico, lo cual se evidencia en el desempeño de la prueba final.

Figura 7. Desempeño cuestionario inicial vs. final.

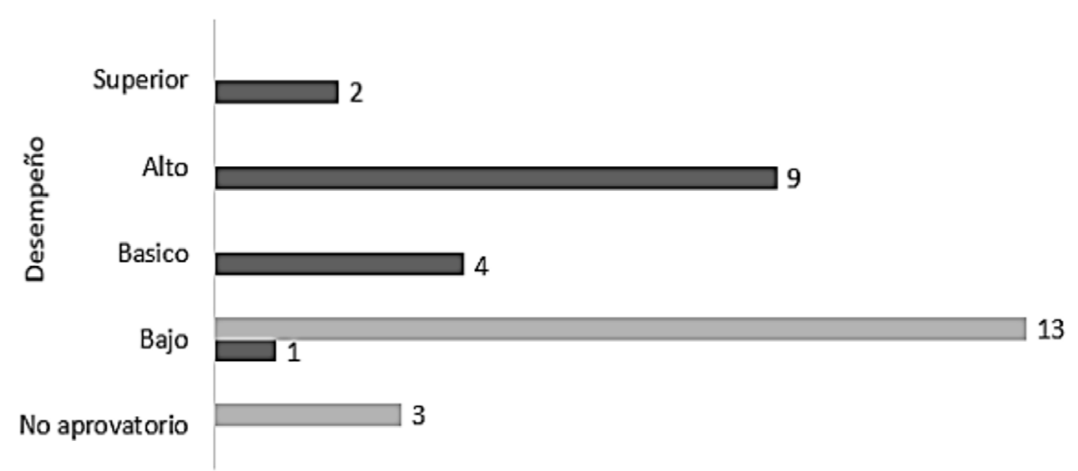

No. Estudiantes

$\square$ Cuestionario Inicial a Cuestionario final

Fuente: elaboración propia.

En la prueba final se observa que quince estudiantes obtuvieron notas aprobatorias, siendo el puntaje mínimo 3.5, como se indicó en la sección "Metodología". En este sentido, los estudiantes en desempeño bajo y no aprobatorio disminuyeron notoriamente, pues de los dieciséis estudiantes evaluados: dos se ubicaron en desempeño superior, nueve lograron posicionarse en un desempeño alto, cuatro se posicionaron en un desempeño básico, y solo un estudiante reprobó la prueba final.

El docente titular de la asignatura manifiesta que "el recurso educativo es innovador y adaptable a la asignatura de tecnología e informática, permitiendo profundizar en los temas de transformación de movimientos, ya que está acorde con los lineamientos del MEN, Ministerio de Educación Nacional, y la guía 30". También 
manifiesta que "es una opción interesante para que los estudiantes construyan su propio conocimiento y lo pongan en práctica, a través de proyectos como la creación de diferentes robots móviles".

\section{Discusión}

A través de la robótica educativa y el uso de referentes pedagógicos y didácticos es posible apoyar los procesos de enseñanza y aprendizaje de la comunidad académica con herramientas tecnológicas (Barrera-Lombana, 2014). Se busca provocar en los estudiantes de educación básica entusiasmo por desarrollar habilidades que les permitan la construcción de saberes, para dar un uso fundamentado, responsable y crítico a la tecnología.

La elaboración de prototipos educativos debe pasar por múltiples etapas de prueba y error. En este caso, para llegar al prototipo final se realizó la simulación de diez versiones del prototipo, hasta llegar al hexápodo funcional. Un proceso similar se evidencia en el trabajo de Araque-Henao y Muriel-Patiño (2018), quienes desarrollaron once versiones, en las cuales probaban: simulaciones, materiales y aplicación, hasta llegar al prototipo funcional.

El uso de prototipos didácticos robóticos en el aula puede ayudar al estudiante a comprender el tema a tratar y su aplicación se da en los diferentes niveles educativos. Ejemplo de ello es el trabajo de Montiel-Jiménez, Córdova-Morales, Paredes-Xochihua y Campos-Peña (2016), quienes construyeron prototipos robóticos para la enseñanza de números a nivel preescolar. En el nivel de educación media se tiene la implementación de una mano robótica didáctica para la enseñanza de los principios de programación en Arduino (Niño-Vega, Martínez-Díaz y Fernández-Morales, 2016). Otro caso es el de Franco y Garnica (2014), con la implementación de robots para fomentar la investigación en ingeniería.

La implementación de estrategias pedagógicas basadas en el AOP permite desarrollar una acción educativa que conlleva los tres 
saberes (Hewitt-Ramírez y Barrero-Rivera, 2012); en este caso: saber, pues el estudiante adquirió nuevos conocimientos sobre el tema de mecanismos; saber hacer, que implica la aplicación de mecanismos ya conocidos para construir el prototipo robótico, llevando los conocimientos a la práctica; y saber ser, donde se genera la interacción entre estudiantes en un ambiente de respeto y colaboración para el desarrollo de proyectos. Según Arellano-Gámez (2009), los tres saberes son la ecuación que en los nuevos tiempos traza y define el camino que nuestros estudiantes deberán recorrer si desean convertirse en profesionales actualizados, competentes y responsables.

La evaluación del aprendizaje tiene gran impacto en la calidad de lo aprendido (Borjas et al., 2019). Por ello, este aspecto debe incluirse en el análisis detallado de los requerimientos técnicos y pedagógicos en cuanto al prototipo del robot hexápodo, identificando los contenidos y el diseño didáctico para la implementación pedagógica del recurso educativo. En cuanto a la aplicación pedagógica del recurso educativo en el aula, se adoptó el enfoque constructivista (Gómez-Pablos, 2018), base del AOP, donde el estudiante siente que lo que aprende está conectado con la realidad construyendo su propio conocimiento.

\section{Conclusiones}

Como resultado del proceso de diseño se obtuvo un prototipo robótico basado en los movimientos que realizan los hexápodos. Estos se aplicaron en un robot educativo que facilita el aprendizaje de diversas temáticas del área de tecnología, especialmente las relacionadas con los conceptos de mecánica.

La construcción del prototipo robótico por parte de los estudiantes, con enfoque en el aprendizaje colaborativo y orientado a proyectos, promueve la interacción y el compañerismo entre estudiantes. 
El trabajo por grupos favorece el aprendizaje de los estudiantes, favoreciendo un trabajo en conjunto. Sin embargo, los resultados indican que la designación de grupos de tres o más estudiantes puede dificultar el desarrollo del proyecto.

Finalmente, se puede afirmar que la aplicación de prototipos didácticos en el aula que lleven al estudiante a la práctica tiene un efecto favorable en su aprendizaje. Esto gracias a que permite relacionar la teoría con la implementación y construcción de diferentes elementos, corroborando su funcionamiento a través de la experimentación.

\section{Referencias bibliográficas}

Alvis-Puentes, J. F., Aldana-Bermúdez, E. y Caicedo-Zambrano, S. J. (2019). Los ambientes de aprendizaje reales como estrategia pedagógica para el desarrollo de competencias matemáticas en estudiantes de básica secundaria. Revista de Investigación, Desarrollo e Innovación, 10(1), 135-147. DOI: https://doi.org/10.19053/20278306.v10.n1.2019.10018

Araque-Henao, L. E. y Muriel-Patiño, C. D. (2018). Diseño y prototipo de una plataforma robótica educativa de manipuladores (tesis de pregrado). Universidad Autónoma de Occidente, Cali, Colombia. Recuperado de https://red.uao.edu.co/bitstream/10614/10353/7/T08025.pdf

Arellano-Gámez, L. A. (2009). La competencia es un saber, saber ser y un saber hacer. Revista Odontológica de Los Andes, 4(1), 3-5. Recuperado de https://imbiomed.com.mx/1/1/articulos.php? method=showDetail\&id_ revista=177\&id_seccion=2993\&id_ejemplar $=6032 \& i d \_a r t i c u l o=59648$

Barrera-Lombana, N. (2014). Uso de la robótica educativa como estrategia didáctica en el aula. Praxis y Saber, 6(11), 215-234. Recuperado de http://www.scielo.org.co/pdf/prasa/v6n11/v6n11a10.pdf

Barrera-Mesa, C. E., Fernández-Morales, F. H. y Duarte, J. E. (2017). Diseño de un ambiente de aprendizaje mediado por TIC para la enseñanza de 
operadores mecánicos orientado al grado séptimo de la educación básica, en el Colegio Boyacá de Duitama. Revista Colombiana de Tecnologías de Avanzada, 2(30), 11-19. DOI: https://doi.org/10.24054/16927257.v30. n30.2017.2740

Borjas, M. P., Navarro-Lechuga. E., Puentes-Ospino, D., De La Cruz-García, J., Yepes-Martínez, J., Muñoz-Alvis, A., Montero, P., De La Hoz-Del Villar, K., Pérez-Moyano, Y. y Polo, J. D. (2019). Experiencias ludoevaluativas en el contexto universitario: la evaluación desde una comunidad de aprendizaje. Revista de Investigación, Desarrollo e Innovación, 10(1), 177-190. DOI: https://doi.org/10.19053/20278306.v10.n1.2019.10021

Bustamante-Pacari, J. (2017). El aprendizaje cooperativo: una competencia imprescindible. Educación Superior, 2(1), 25-36. Recuperado de http://www.scielo.org.bo/scielo.php?script=sci_arttext\&pid=S2518$82832017000100003 \& \operatorname{lng}=e s \&$ tlng $=e s$

Cabrera, J., Sánchez, I., Rojas, F. y Arias-Rojas, J. (2017). Prototipo de guía didáctica para la enseñanza - aprendizaje de la física en ingeniería mediada por herramientas digitales disponibles en la web: uso de simuladores. IV Congreso Internacional AmITIC (pp. 132-141).

Recuperado https://revistas.utp.ac.pa/index.php/memoutp/article/ view/1481

Cárdenas-Martínez, L. D. (2019). La creatividad y la educación en el siglo XXI. RIIEP: Revista Interamericanade Investigación, Educación y Pedagogía, 12(2), 211-224. DOI: https://doi.org/10.15332/25005421.5014

Carrera, L. y Ramírez, M. (2017). Diseño, implementación e impacto de prototipos experimentales para mejorar la enseñanza de la ley de BiotSavart en estudiantes de ingeniería. Latin-American Journal of Physics Education, 11(2), 1-8. Recuperado de https://dialnet.unirioja.es/servlet/ articulo? codigo $=6353423$

Cruz-Rojas, G. A., Molina-Blandón, M. A. y Valdiri-Vinasco, V. (2019). Vigilancia tecnológica para la innovación educativa en el uso de bases de datos y plataformas de gestión de aprendizaje en la Universidad del 
Valle, Colombia. Revista de Investigación, Desarrollo e Innovación, 9(2), 303-317. DOI: https://doi.org/10.19053/20278306.v9.n2.2019.9175

Franco, J. y Garnica, E. (2014). Enseñanza en las aulas de clase con robots y el fomento de la investigación en ciencias e ingeniería. En Memorias del Congreso Iberoamericano de Ciencia, Tecnología, Innovación y Educación (Buenos Aires, Argentina) (art. 893). Recuperado de https:// www.oei.es/historico/congreso2014/contenedor.php?ref=memorias

Gómez-Pablos, V. B. (2018). El valor del aprendizaje basado en proyectos con tecnologías: análisis de prácticas de referencia (tesis de doctorado). Instituto Universitario de Ciencias de la Educación, Salamanca, España. Recuperado de https://bechallenge.io/uploads/videos/1555937706.pdf

González, D., Estrada, E. y Roldán, J. (2016). Aplicación Android para el estudio de mecanismos planos de cuatro barras. Entre Ciencia $e$ Ingeniería, 10(20), 41-51. Recuperado de http://www.scielo.org.co/ scielo.php?script=sci_arttext \&pid=S1909-83672016000200007\&lng=es \&tlng=es

González-Campos, D., Olarte-Dussán, F. y Corredor-Aristizábal, J. (2017). La alfabetización tecnológica: de la informática al desarrollo de competencias tecnológicas. Estudios Pedagógicos, 43(1), 193-212. DOI: http://dx.doi.org/10.4067/S0718-07052017000100012

Gutiérrez, C. A. (2018). Herramienta didáctica para integrar las TIC en la enseñanza de las ciencias. RIIEP: Revista Interamericana de Investigación, Educación y Pedagogía, 11(1), 101-126. DOI: https://doi.org/10.15332/ s1657-107X.2018.0001.03

Gutiérrez-Rico, D., Almaraz-Rodríguez, O. D. y Bocanegra-Vergara, N. (2019). Concepciones del docente en sus formas de percibir el ejercicio de la investigación desde su práctica. Revista de Investigación, Desarrollo e Innovación, 10(1), 149-161. DOI: https://doi.org/10.19053/20278306. v10.n1.2019.10019

Herrera-Baquero, L. P. y Prieto-Ortiz, F. A. (2018). Metodología para la inspección de la herramienta en el taladrado de piezas. Revista de 
Investigación, Desarrollo e Innovación, 9(1), 187-200. DOI: https://doi. org/10.19053/20278306.v9.n1.2018.7937

Hewitt-Ramírez, N. y Barrero-Rivera, F. (2012). La integración de los saberes: una propuesta curricular para la formación en investigación en la educación superior. Psychologia: Avances de la Disciplina, 6(1), 137-145. Recuperado de https://dialnet.unirioja.es/servlet/ articulo? codigo $=4924046$

Jiménez-Espinosa, A. (2019). La dinámica de la clase de matemáticas mediada por la comunicación. Revista de Investigación, Desarrollo e Innovación, 10(1), 121-134. DOI: https://doi.org/ 10.19053/20278306. v10.n1.2019.10016

Machado, D., Herrera, G., Roldán, J. y Díaz, J. (2015). Una herramienta computacional didáctica para el análisis cinemático de mecanismos planos de cuatro barras. UIS Ingenierías, 14(1), 59-69. Recuperado de https://revistas.uis.edu.co/index.php/revistauisingenierias/article/ view/59-69/4981

Maldonado-Pérez, M. (2008). Aprendizaje basado en proyectos colaborativos: una experiencia en educación. Laurus, 14(28), 158-180. Recuperado de https://www.redalyc.org/articulo.oa?id=76111716009

Márquez, J.-E. y Ruiz, J.-H. (2014). Robótica educativa aplicada a la enseñanza básica secundaria. Didáctica, Innovación y Multimedia, 10(30), 1-12. Recuperado de: https://dialnet.unirioja.es/servlet/ articulo?codigo $=5081638$

Martínez, C. (2005). Introducción a los escarabajos Carabidae (Coleoptera) de Colombia. Bogotá: Instituto de Investigación de Recursos Biológicos Alexander von Humboldt.

Montiel-Jiménez, E., Córdova-Morales, G., Paredes-Xochihua, F. y Campos-Peña, J. (2016). Diseño y construcción de un prototipo de robot educativo para apoyo de la enseñanza de los números a nivel preescolar. La Mecatrónica en México, 5(2), 47-55. Recuperado de http://www. mecamex.net/revistas/LMEM/revistas/LMEM-V05-N02-02.pdf 
Mosquera-Mosquera, C. E. y Ramírez-Martínez, J. E. (2020). El desencantamiento de la escuela: sus cambios y zarandeos en la era posindustrial. RIIEP: Revista Interamericana de Investigación, Educación y Pedagogía, 13(1), 45-61. Recuperado de https://revistas.usantotomas. edu.co/index.php/riiep/article/view/5459

Niño-Vega, J. A. y Fernández-Morales, F. H. (2019). Una mirada a la enseñanza de conceptos científicos y tecnológicos a través del material didáctico utilizado. Revista Espacios, 40(15), art. 4. Recuperado de http://www.revistaespacios.com/a19v40n15/19401504.html

Niño-Vega, J. A., Fernández-Morales, F. H. y Duarte, J. E. (2019). Diseño de un recurso educativo digital para fomentar el uso racional de la energía eléctrica en comunidades rurales. Saber, Ciencia y Libertad, 14(2), 256272. DOI: https://doi.org/10.18041/2382-3240/saber.2019v14n2.5889

Niño-Vega, J. A., Martínez-Díaz, L. Y. y Fernández-Morales, F. H. (2016). Mano robótica como alternativa para la enseñanza de conceptos de programación en Arduino. Revista Colombiana de Tecnologías de Avanzada, 2(28), 132-139. DOI: https://doi.org/10.24054/16927257. v28.n28.2016.2476

Ñáñez-Rodríguez, J. J., Solano-Guerrero, J. C. y Bernal-Castillo, E. (2019). Ambientes digitales de aprendizaje en educación a distancia para la formación inicial de docentes: percepciones acerca de su pertinencia. Revista de Investigación, Desarrollo e Innovación, 10(1), 107-119. DOI: http://doi.org/10.19053/20278306.v10.n1.2019.10015

Ordóñez-Ortega, O., Gualdrón-Pinto, E. y Amaya-Franky, G. (2019). Pensamiento variacional mediado con baldosas algebraicas y manipuladores virtuales. Revista de Investigación, Desarrollo e Innovación, 9(2), 347-362. DOI: https://doi.org/10.19053/20278306. v9.n2.2019.9180

Ortega, J.-J. y Sigut, M. (2016). Prototipo de una plataforma móvil de bajo coste para simulación de vuelo de alto realismo. Revista Iberoamericana de Automática e Informática Industrial (RIAI), 13(3), 293-303. Recuperado de https://dialnet.unirioja.es/servlet/articulo?codigo=6016249 
Rico-Martínez, J. (2019). Análisis de un mecanismo de manivela biela corredera para propósitos de balanceo de maquinas de combustión interna. Recuperado de http://www.dicis.ugto.mx/profesores/chema/documentos/Din\%C3\%A1mica\%20de\%20Maquinaria/AnalisisMecanismoBMCCombustionInterna.pdf

Ruiz-Macías, E. y Duarte, J. E. (2018). Diseño de un material didáctico computarizado para la enseñanza de oscilaciones y ondas, a partir del estilo de aprendizaje de los estudiantes. Revista de Investigación, Desarrollo e Innovación, 8(2), 295-309. DOI: https://doi.org/10.19053/20278306. v8.n2.2018.7966

Tamayo, O. E., Zona, R. y Loaiza, Y. E. (2015). El pensamiento crítico en la educación: algunas categorías centrales en su estudio. Revista Latinoamericana de Estudios Educativos, 11(2), 111-133. Recuperado de https://www.redalyc.org/articulo.oa?id=134146842006

Todd, P., Mueller, D. y Fichter, E. (2014). Atlas of the four-bar linkage (2nd ed.). Tigard: Saltire Software.

Torres-Barahona, E., León-Medina, J. y Pinto-Salamanca, M. (2017). Metodología de análisis de mecanismos y uso de software para su implementación. XI Congreso Colombiano de Métodos Numéricos. HAL id: hal-01715664.

Tovar-Vergara, E. L. (2019). Implementación de estrategias pedagógicas constructivistas mediadas por las herramientas web 2.0 para el fortalecimiento de la comprensión teórica en los contenidos conceptuales de las ciencias naturales y la educación ambiental. RIIEP: Revista Interamericana de Investigación, Educación y Pedagogía, 12(2), 71-112. DOI: https://doi.org/10.15332/25005421.5009

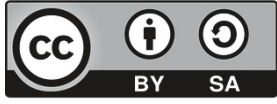

